\title{
Arquiteturas Pedagógicas na Formação de Professores: Tecnologias Educacionais, Extensão Universitária e Ensino de Língua Portuguesa
}

\author{
Marcelo Rocha Barros Gonçalves ${ }^{1}$, Juliana Wolf Pereira ${ }^{1}$, \\ Viviane Teles ${ }^{1}$, Justina Souza Souto Paiva ${ }^{2}$ \\ ${ }^{1}$ Universidade Federal de Mato Grosso do Sul \\ Campus de Coxim (CPCX) - Coxim - MS - Brasil \\ ${ }^{2}$ Coordenadoria Regional de Educação - CRE 4 \\ Secretaria de Educação do Estado - Coxim - MS - Brasil \\ \{marcelo.barros\}@ufms.br
}

\begin{abstract}
In this work, a report about the development, provision and evaluation of Digital Information and Communication Technologies (TDIC), in a University Extension Projects, is presented. The study deals with the design and implementation of TDIC, its applications and the analysis of results in the course "Textual Genres in the Portuguese Language Olympiad", held between the University and public schools, within a perspective of construction of Pedagogical Architectures for teacher training. The use of TDIC's promoted a high degree of satisfaction among the audience, in this case, both teachers and students. This result reinforces the importance of educational public policies based on TDIC, due to the positive impact of the use of technology in teaching-learning processes.
\end{abstract}

Resumo. Este trabalho apresenta um relato de experiência do desenvolvimento, disponibilização e avaliação de tecnologias digitais da informação e comunicação (TDIC) em projetos de extensão universitária. O estudo em questão trata da concepção e implementação de TDIC, suas aplicações e análise de seus resultados no Curso de Extensão Gêneros Textuais na Olimpíada de Língua Portuguesa, realizado entre a Universidade Federal de Mato Grosso do Sul (UFMS) e escolas públicas, dentro da perspectiva de Arquiteturas Pedagógicas para a formação de professores. Com a avaliação das TDICs em ações de extensão, os resultados mostraram alto grau de satisfação do público alvo atingido, neste caso professores-alunos. Isso reforça o impacto positivo do uso da tecnologia aplicada ao processo de ensino e aprendizagem em ações de políticas públicas para a sociedade.

\section{Introdução}

É consensual que a educação superior está alicerçada em três grandes pilares: o ensino, a pesquisa e a extensão. Na extensão universitária há o compartilhamento entre o conhecimento científico e tecnológico produzido na universidade e os conhecimentos de que são titulares as comunidades tradicionais [de Paula 2013]. Não são raros os casos em que são utilizadas tecnologias digitais para o desenvolvimento de ações de extensão nas mais diversas áreas do conhecimento. Listamos, por exemplo, o trabalho com TDIC na extensão 
VII Congresso Brasileiro de Informática na Educação (CBIE 2018)

Anais dos Workshops do VII Congresso Brasileiro de Informática na Educação (WCBIE 2018)

universitária para professores de matemática [Javaroni and Zampieri 2015] e na Universidade Aberta à Terceira Idade [Lamarca et al. 2015]. Vale lembrar também do trabalho Práticas pedagógicas para ciberinfância: um desafio atual [do Amaral et al. 2010] no qual as autoras apresentam hipóteses sobre o desafio de educar na contemporaneidade a partir da realização de cursos de extensão com apoio de artefatos tecnológicos digitais para educadores dos anos iniciais.

O presente artigo é um relato de experiência de um curso de extensão realizado em parceria entre a Universidade Federal de Mato Grosso do Sul (UFMS) e a Coordenadoria Regional de Educação CRE 4 (Secretaria de Educação do Estado) com vistas à formação docente de professores da rede pública da região norte do Mato Grosso do Sul, realizado no segundo semestre de 2017, com foco especial no ensino e aprendizagem mediados por computador e no ensino de conteúdos específicos enriquecidos por tecnologias digitais de informação e comunicação (TDIC), utilizando como pano de fundo a Olimpíada de Língua Portuguesa (OLP).

O programa da OLP é uma ação da Fundação Itaú Cultural com coordenação técnica do Centro de Estudos e Pesquisas em Educação, Cultura e Ação Comunitária (CENPEC). No portal, disponível em www. escrevendoofuturo.org, os usuários tem acesso ao programa, bem como a uma série de outras funções, como cursos online, biblioteca virtual e vídeos para formação de professores, com experiências didáticas, relatos de prática docente e palestras.

Nossa perspectiva compreende a utilização de TDIC a partir da construção de Arquiteturas Pedagógicas para a formação de professores, tomando os indivíduos como protagonistas de seu próprio desenvolvimento para a aquisição de habilidades e competências. A formação de professores que estão em sala de aula e se capacitando ao mesmo tempo, respeitando assim seu duplo pertencimento, transforma em atores da cena pedagógica o mesmo sujeito, ou seja, um professor-aluno.

Neste trabalho faremos a apresentação do resultado da pesquisa realizada com 62 professores da rede pública da região norte do Mato Grosso do Sul, participantes do curso de extensão "Genêros Textuais na Olimpíada de Língua Portuguesa".

\section{Fundamentação Teórica}

O uso de TDIC em ambientes escolares tem permitido a utilização de novas práticas e métodos no processo de ensino e aprendizagem. Para [Sarmento et al. 2017] a formação do professor deve estar elencada sobre as possibilidades e maneiras de se utilizar tais recursos em prol de uma prática pedagógica efetiva. Os autores [Andrade and Ferrete 2017] percebem que a tecnologia no ambiente escolar lança novos desafios, exigindo uma estrutura física adequada, que proporcione acesso de qualidade a rede e formação de professores e gestores, o que entendem como uma cultura escolar.

A utilização e desenvolvimento de ambientes virtuais de aprendizagem (AVA) e de recursos educacionais abertos (REA) tem se tornado fonte de estudo para inúmeros grupos de pesquisa, além de permitir a democratização, universalização e acesso ao conhecimento.

Um AVA é um espaço interativo e dinâmico, que possibilita a disponibilização de diferentes recursos de mídia e permite um processo de ensino e aprendizagem co- 
laborativo, criativo e interessante. O termo recursos educacionais abertos (REA), mais conhecido pela expressão em inglês Open Educational Resources (OER), apareceu pela primeira vez em uma conferência da UNESCO em 2002. REA abrange qualquer material educativo, tais como, cursos, módulos de conteúdo, objetos de aprendizagem entre outros. REA são materiais digitais educacionais de forma livre e aberta para uso no ensino, aprendizagem e pesquisa, para toda a comunidade acadêmica [Hylén 2006], [Richter and McPherson 2012].

A abordagem de ensino Blended Learning possibilita o uso de recursos de educação a distância e presencial num processo de ensino e aprendizagem [Bacich 2016]. Conforme [Garrison and Kanuka 2004] e [Ramos et al. 2014], a experiência educacional promovida pela integração do ensino presencial e de recursos tecnológicos como AVA e REA, permite o enriquecimento e amplia a construção do conhecimento colaborativo. Respeitamos assim, conforme [Novak et al. 2014], que uma arquitetura pedagógica deve se alicersar numa concepção pedagógica forte e embasada, numa sistematização metodológica do trabalho realizado e com o suporte telemático.

Também consideramos importantes neste trabalho, como fazem [do Amaral et al. 2010], definir com cuidado os aspectos instrucionais e metodológicos, com especial atenção à construção de objetos de aprendizagem. Na área de Língua Portuguesa, os gêneros textuais, dentro da ótica de [Schneuwly and Dolz-Mestre 2004], são instrumentos culturais disponíveis nas interações sociais. São historicamente mutáveis e, consequentemente, relativamente estáveis. Emergem em diferentes domínios discursivos e se concretizam em textos, que são singulares.

Os Parâmetros Curriculares Nacionais (PCN) de Língua Portuguesa estão sustentados, sobretudo, na teoria dos gêneros textuais, propondo que o trabalho com a língua materna, no que concerne ao ensino dos patrimônios significativos da linguagem, tanto no que se refere ao contexto oral quanto ao escrito, amplie o conhecimento necessário para que os sujeitos envolvidos nos processos de ensino e aprendizagem saibam ajustar suas práticas linguísticas, com êxito, aos eventos sociais comunicativos de que já participam e que ainda irão participar. O labor com a Língua Portuguesa, por conseguinte, deve projetar a ampliação das várias perspectivas do uso da linguagem, em qualquer forma de prática.

\section{Metodologia}

O curso foi realizado em parceria entre a UFMS e a Coordenadoria Regional de Educação utilizando um método híbrido no qual os sujeitos participantes representam o público alvo desta análise.

As atividades foram desenvolvidas na modalidade presencial para o município sede e semi presencial (webconferência) para as cidades jurisdicionadas, com atividades de ensino a distância (EAD) utilizando o Moodle.

O curso foi dividido em quatro módulos, correspondentes aos quatro gêneros utilizados para a produção dos textos na OLP: poema/poesia, memória, crônica e artigo de opinião.

Em todos os módulos, utilizamos os seguintes eixos temáticos para a discussão:

- Eixo I - Reflexão sobre Gêneros Textuais: conceito e finalidade; adequação aos 
VII Congresso Brasileiro de Informática na Educação (CBIE 2018)

Anais dos Workshops do VII Congresso Brasileiro de Informática na Educação (WCBIE 2018)

critérios estabelecidos para a Olimpíada de Língua Portuguesa.

- Eixo II - Utilização do livro didático como suporte para o estudo do gênero no cotidiano de alunos e professores.

- Eixo III - Oficina para embasar a prática docente do gênero estudado em sala de aula.

Nas tabelas 1, 2, 3 e 4 , pode ser observado o conteúdo estudado em cada módulo.

Os professores-alunos puderam avaliar o curso através de um questionário Google Forms, quanto à adequação de conteúdo e carga horária, quanto à utilização do material das aulas, dentre outras. Com o formulário foi possível também traçar melhor o perfil dos professores da rede e suas habilidades e competências no uso das tecnologias. De maneira geral, a resposta dos professores-alunos foi bastante positiva. Além da ampla participação no questionário (59 de 62 alunos), o curso e os conteúdos apresentados foram bem avaliados.

\section{Análise dos Resultados}

Nesta seção são apresentados os resultados obtidos na análise dos dados gerados com as respostas de um questionário aplicado aos participantes do curso. O instrumento utilizado para avaliação foi elaborado pelos autores. A escala de Likert foi utilizada pois permite respostas que variam em gradiência do mais alto grau de satisfação ao mais baixo. Neste trabalho, a variação da escala consiste em:
A - Concordo plenamente
B - Concordo parcialmente
C - Não concordo nem discordo
D - Discordo parcialmente
E - Discordo plenamente

A oferta do curso permitiu realizar um levantamento sobre a área de formação e o nível de formação dos professores da rede pública da região, conforme pode ser observado na figura 1 .

Assim como detectou [Carvalho et al. 2005] o público também foi eminentemente feminino, totalizando 93,2\% dos participantes. Foi possível verificar, também, o tempo de docência dos professores-alunos. Apenas $1 / 4$ dos professores-alunos $(25,5 \%)$ atuam há menos de dez anos na docência. O conteúdo fornecido pelo curso foi avaliado pelos professores-alunos quanto à sua relevência e aplicabilidade, conforme pode ser observado na figura 2.

Os professores-alunos puderam avaliar a interação com o Ambiente Moodle, considerado como fácil de usar, interativo e visualmente atrativo. A utilização do Moodle se mostrou eficaz para a execução da ação de extensão, conforme pode ser observado na figura 3.

Para apenas 3,4 \% dos participantes, os conhecimentos adquiridos no curso não seriam aplicáveis em suas rotinas de trabalho.

\section{Considerações Finais}

Este trabalho apresentou um relato de experiência da utilização de TDIC para o Curso de Extensão. Para o curso foram desenvolvidas atividades presenciais e atividades num am- 
VII Congresso Brasileiro de Informática na Educação (CBIE 2018)

Anais dos Workshops do VII Congresso Brasileiro de Informática na Educação (WCBIE 2018)

Tabela 1. Conteúdo do módulo I

\begin{tabular}{|c|c|c|}
\hline Módulo & Gênero Textual & Conteúdo \\
\hline I & Poesia/Poema & $\begin{array}{l}\text { Material de leitura obrigatória: } \\
\text { - Cidadezinha de Mário Quintana (leitura/ } \\
\text { vídeo e audição); } \\
\text { - As Marias do meu lugar de Carlos Victor } \\
\text { Dantas Araújo; } \\
\text { - Haicai de Guilherme de Almeida. } \\
\text { Material de leitura complementar: } \\
\text { - Cidadezinha Qualquer de Carlos Drum- } \\
\text { mond de Andrade leitura/ vídeo e audição); } \\
\text { - História do cordel; } \\
\text { - Convite de José Paulo Paes; } \\
\text { - Duas dúzias de coisinhas à toa que deixam } \\
\text { a gente feliz de Otávio Roth. } \\
\text { Descritores avaliativos; } \\
\text { Atividade do módulo: redigir uma poesia com } \\
\text { base nas imagens e palavras apresentadas nos sli- } \\
\text { des do PowerPoint da atividade. }\end{array}$ \\
\hline
\end{tabular}

Tabela 2. Conteúdo do módulo II

\begin{tabular}{|c|c|c|}
\hline Módulo & Gênero Textual & Conteúdo \\
\hline II & Memórias & $\begin{array}{l}\text { Material de leitura obrigatória: } \\
\text { - Memórias João Acaiabe - vídeo; } \\
\text { - O Valentão que engolia Meninos e Outras } \\
\text { Histórias de Pajé; } \\
\text { - Artigo: Memória, rememoração e lem- } \\
\text { brança em Maurice Halbwachs de Luana } \\
\text { Aparecida Matos Leal. } \\
\text { Material de leitura complementar: } \\
\text { - Memórias - gênero em pauta - vídeo; } \\
\text { - Lembranças que o tempo não apagou - áu- } \\
\text { dio; } \\
\text { - Memórias literárias - Nas ruas do Brás de } \\
\text { Drauzio Varella; } \\
\text { Descritores avaliativos; } \\
\text { Atividade do módulo: redigir um texto que re- } \\
\text { meta às memórias do lugar onde vive. }\end{array}$ \\
\hline
\end{tabular}


VII Congresso Brasileiro de Informática na Educação (CBIE 2018)

Anais dos Workshops do VII Congresso Brasileiro de Informática na Educação (WCBIE 2018)

Tabela 3. Conteúdo do módulo III

\begin{tabular}{|c|c|c|}
\hline Módulo & Gênero Textual & Conteúdo \\
\hline III & Crônicas & $\begin{array}{l}\text { Material de leitura obrigatória: } \\
\text { - Engenheiros do hawaí - vídeo; } \\
\text { - Amor acaba de Paulo Mendes Campos; } \\
\text { - Cobrança de Moacy Scliar; } \\
\text { Material de leitura complementar: } \\
\text { - Meu último desejo de Arnaldo Jabor; } \\
\text { - Entre amigos de Martha Medeiros; } \\
\text { - Crônica do Amor Arnaldo Jabor - vídeo; } \\
\text { Descritores avaliativos; } \\
\text { Atividade do módulo: redigir uma crônica sobre } \\
\text { o "Lugar onde vive". }\end{array}$ \\
\hline
\end{tabular}

Tabela 4. Conteúdo do módulo IV

\begin{tabular}{|c|c|c|}
\hline Módulo & Gênero Textual & Conteúdo \\
\hline IV & $\begin{array}{l}\text { Artigo de Opi- } \\
\text { nião }\end{array}$ & $\begin{array}{l}\text { Material de leitura obrigatória: } \\
\text { - Doce Içara com sabor amargo de Joyce } \\
\text { Silva - vídeo; } \\
\text { Elementos articuladores / o que é argu- } \\
\text { mentar; } \\
\text { - O gênero artigo de opinião: da teoria à } \\
\text { prática em sala de aula de Marcia Cristina } \\
\text { Greco e Francimara de Souza Barbosa; } \\
\text { Material de leitura complementar: } \\
\text { - Em defesa do voto obrigatório de Léo } \\
\text { Lince; } \\
\text { - Aula de Artigo de opinião - vídeos do You- } \\
\text { tube; } \\
\text { - Fontes confiáveis na Internet - Vídeo do } \\
\text { site escrevendo o futuro; } \\
\text { - A estrutura do argumento - esquema de } \\
\text { Toulmin; } \\
\text { - Endereços de vídeos relacionados ao Ar- } \\
\text { tigo de Opinião; } \\
\text { Descritores avaliativos; } \\
\text { Atividade do módulo: redigir um artigo de opi- } \\
\text { nião sobre um assunto do "Lugar onde vive". }\end{array}$ \\
\hline
\end{tabular}


VII Congresso Brasileiro de Informática na Educação (CBIE 2018)

Anais dos Workshops do VII Congresso Brasileiro de Informática na Educação (WCBIE 2018)

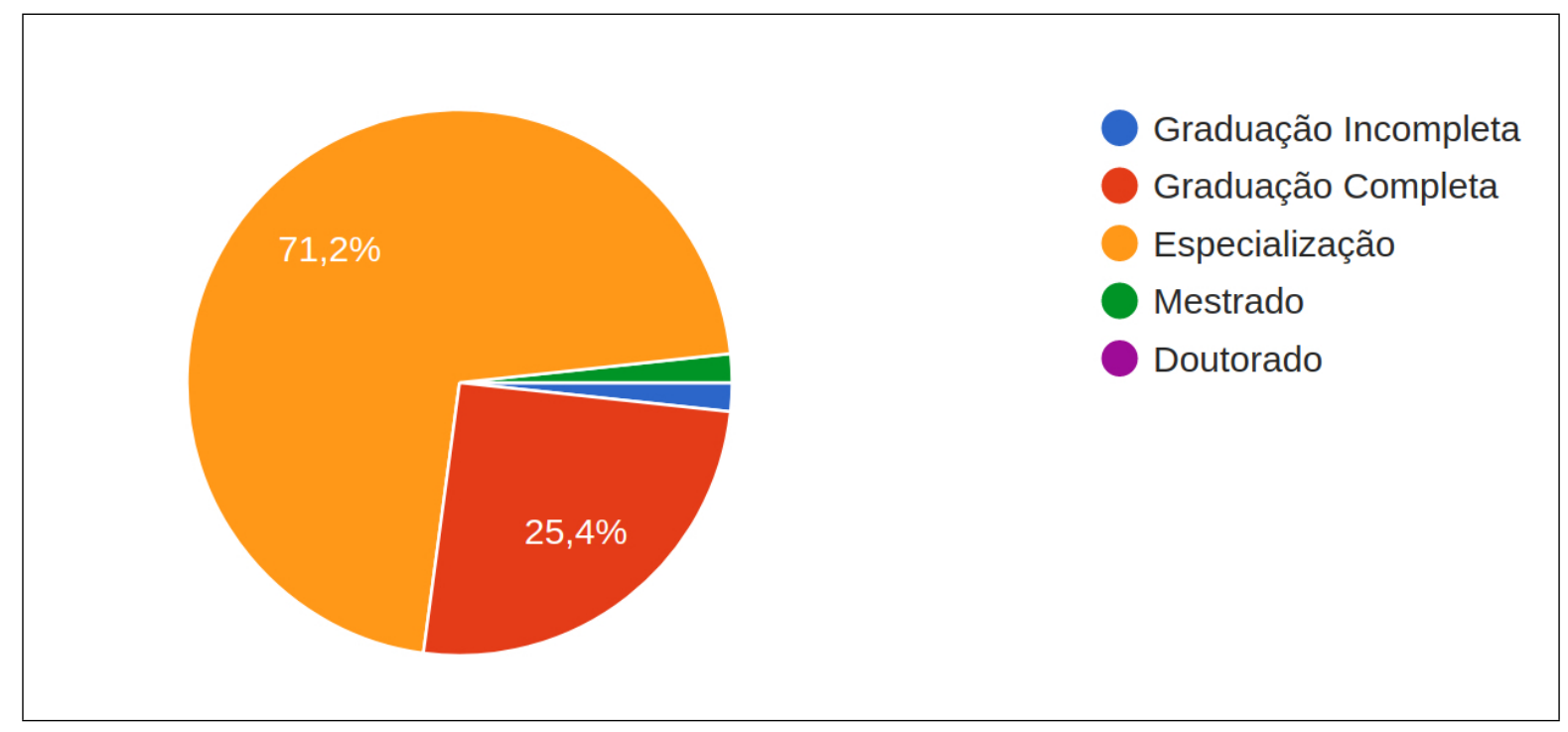

Figura 1. Nível de formação

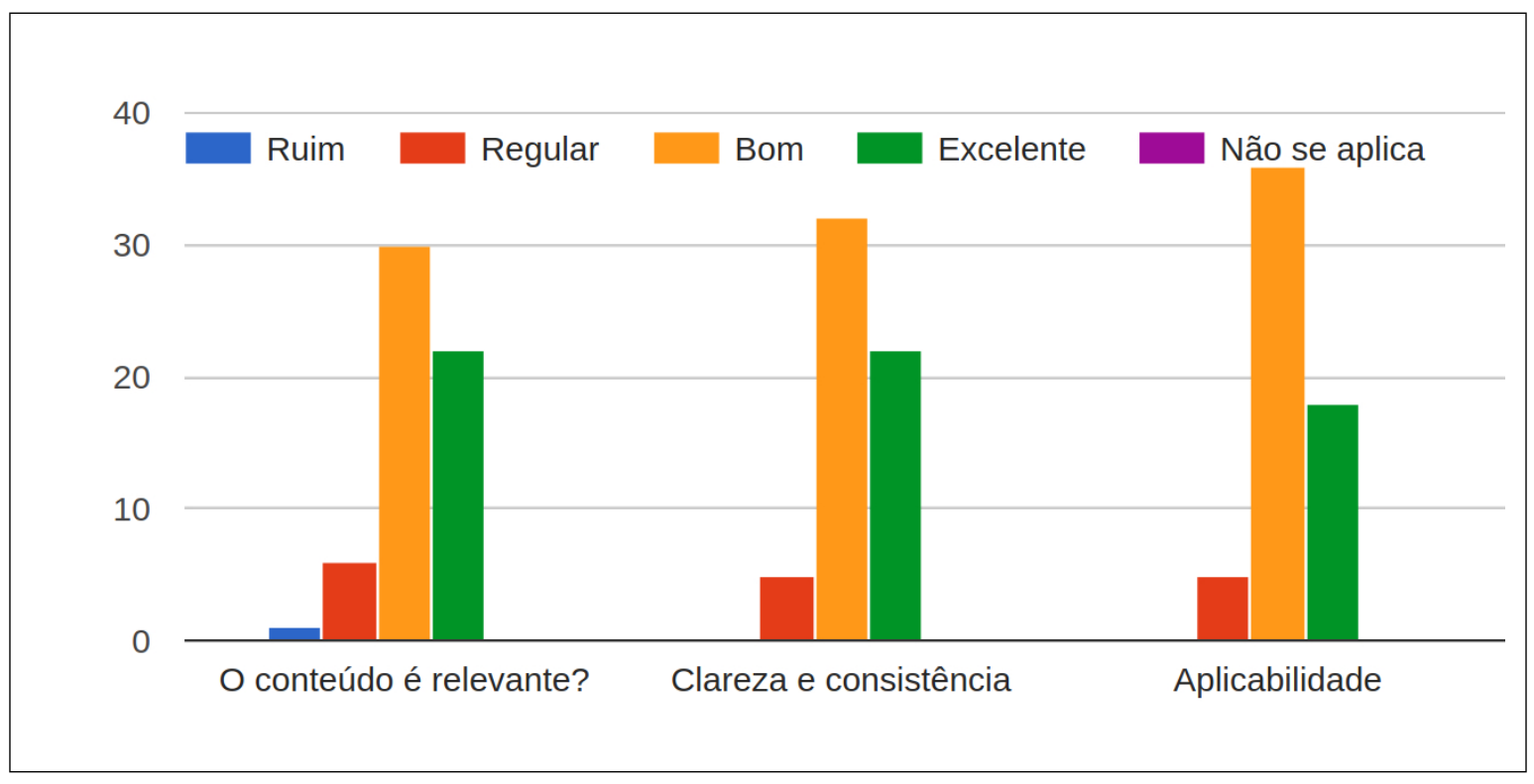

Figura 2. Conteúdo disponível no Moodle

biente virtual de aprendizagem, síncronas e assíncronas. No que tange o AVA, confirma-se a necessidade de feedback aos alunos (usuários) conforme [Costa et al. 2016].

O uso de TDIC permitiu repensar o processo de ensino e aprendizagem, seja do ponto de vista estrutural, seja do ponto de vista da formação dos professores. Assim, tanto as escolas como o corpo docente devem estar preparados para a utilização de TDIC no ambiente escolar [Soares et al. 2016].

Percebemos ainda que devem-se aprimorar a coleta de dados, bem como a análise dos resultados. A análise dos resultados, por sua vez, deve ser realizada em conssonância com os dados já produzidos pelo Plano Estadual de Educação e os respectivos Planos Municipais de Educação. 
VII Congresso Brasileiro de Informática na Educação (CBIE 2018)

Anais dos Workshops do VII Congresso Brasileiro de Informática na Educação (WCBIE 2018)

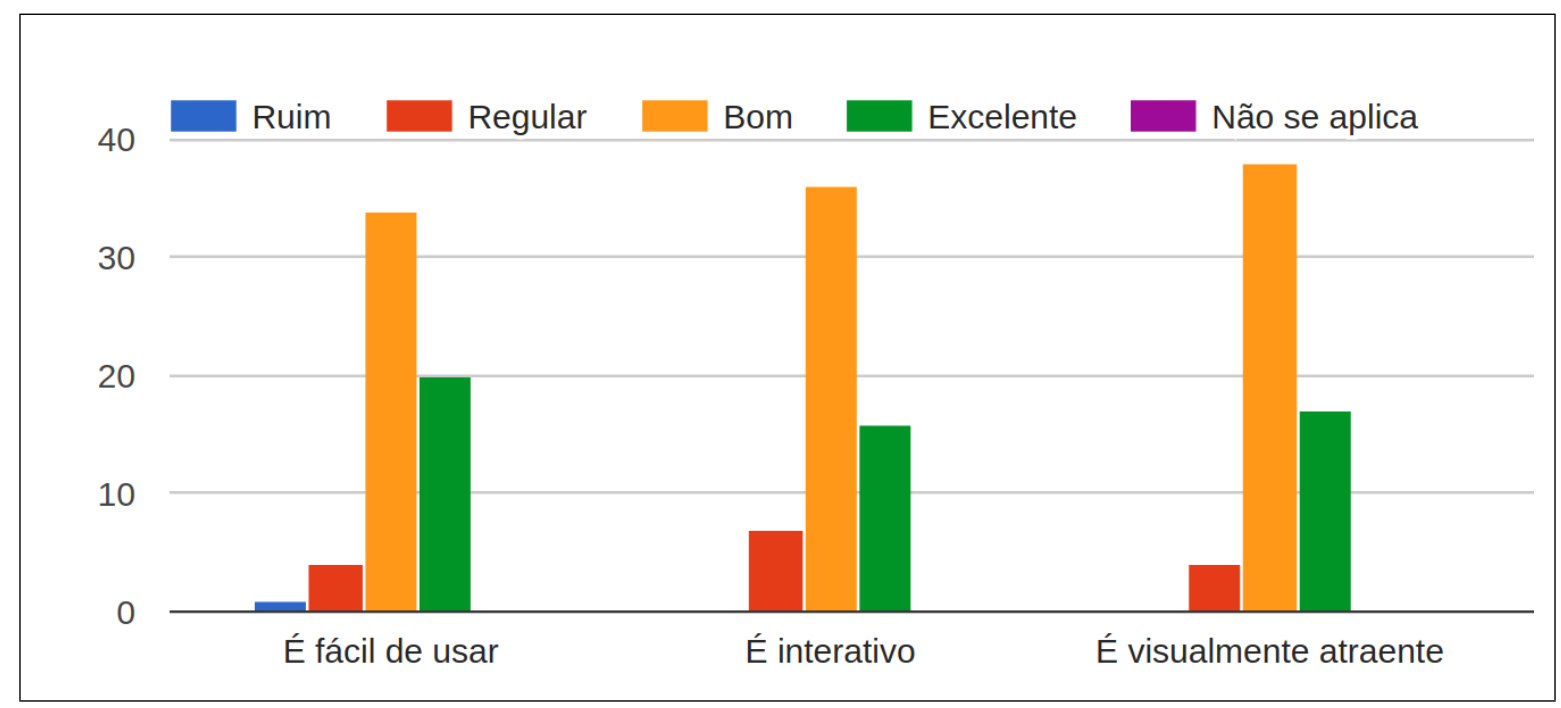

Figura 3. Interação com o ambiente virtual

A avaliação do trabalho foi realizada através da análise dos questionários preenchidos pelos participantes do projeto. As questões tratavam sobre o conhecimento em informática e em EAD, sobre o conteúdo disponibilizado no AVA e sobre a interação com o AVA. Os conteúdos foram considerados relevantes, contextualizados e de qualidade. Foi ainda indicada a facilidade e interatividade com o AVA. Um dos itens a ser aprimorado é a interface com o usuário que deve ser visualmente mais atraente.

As atividades propostas no ambiente virtual foram consideradas relevantes e replicáveis, bem como o método foi considerado interessante. Lamentavelmente, verificamos que a estrutura das escolas ainda está aquém da exigida para a utilização de TDIC.

Vale destacar que o uso de TDIC em ações de extensão da Universidade permite um alcance geográfico maior, priorizando e evidenciando o caráter universal e democrático do conhecimento desenvolvido nas instituições de ensino superior no país.

Para os trabalhos futuros pretende-se aprimorar o AVA, desenvolver novos REA e executar a proposta em outras escolas.

\section{Referências}

Andrade, C. and Ferrete, A. A. S. S. (2017). Formação docente: percepções dos professores sobre o uso das tecnologias móveis digitais no processo de ensino e aprendizagem. In Anais dos Workshops do Congresso Brasileiro de Informática na Educação, volume 6, page 515 .

Bacich, L. (2016). Ensino híbrido: Proposta de formação de professores para uso integrado das tecnologias digitais nas ações de ensino e aprendizagem. In Anais do Workshop de Informática na escola, volume 22, page 679.

Carvalho, M. J. S., de Nevado, R. A., and de Menezes, C. S. (2005). Arquiteturas pedagógicas para educação à distância: concepções e suporte telemático. In Brazilian Symposium on Computers in Education (Simpósio Brasileiro de Informática na EducaçãoSBIE), volume 1, pages 351-360. 
VII Congresso Brasileiro de Informática na Educação (CBIE 2018)

Anais dos Workshops do VII Congresso Brasileiro de Informática na Educação (WCBIE 2018)

Costa, E., Fechine, J., Silva, P., and Rocha, H. (2016). Modelos de feedback para estudantes em ambientes virtuais de aprendizagem. Jornada de Atualização em Informática na Educação, 5(1):1-38.

de Paula, J. A. (2013). A extensão universitária: história, conceito e propostas. InterfacesRevista de Extensão da UFMG, 1(1):5-23.

do Amaral, C. B., Behar, P. A., and Dornelles, L. V. (2010). Práticas pedagógicas para ciberinfância: um desafio atual. In Brazilian Symposium on Computers in Education (Simpósio Brasileiro de Informática na Educação-SBIE), volume 1.

Garrison, D. R. and Kanuka, H. (2004). Blended learning: Uncovering its transformative potential in higher education. The internet and higher education, 7(2):95-105.

Hylén, J. (2006). Open educational resources: Opportunities and challenges. Proceedings of Open Education, pages 49-63.

Javaroni, S. L. and Zampieri, M. T. (2015). O uso das tic nas práticas dos professores de matemática da rede básica de ensino: o projeto mapeamento e seus desdobramentos. Bolema: Boletim de Educação Matemática, 29(53):998-1022.

Lamarca, D. S. F., Pires, L. F., Vieira, S. C., and Lourenzani, A. E. S. B. (2015). Tic presente nas universidades: uma análise no projeto de extensão universidade aberta à terceira idade (unati). In Congresso de extensão universitária da UNESP, pages 1-8. Universidade Estadual Paulista (UNESP).

Novak, S., Aragón, R., Ziede, M. K. L., and Menezes, C. S. d. (2014). Aprendizagem em rede na educação a distância: práticas e reflexões. Evangraf.

Ramos, J. L. C., da Silva, R. F. P., Silva, J. C. S., and Gomes, A. S. (2014). Adoção de blended learning: verificação do potencial de ampliação na universidade federal do vale do são francisco. In Anais do Workshop de Informática na Escola, volume 20, page 36 .

Richter, T. and McPherson, M. (2012). Open educational resources: education for the world? Distance education, 33(2):201-219.

Sarmento, A., Farias, M., and Santos, H. (2017). Relato de experiência do pibid: Promovendo o ensino de computação de forma interdisciplinar com português no ensino fundamental. In Anais do Workshop de Informática na Escola, volume 23, page 313.

Schneuwly, B. and Dolz-Mestre, J. (2004). Gêneros orais e escritos na escola.

Soares, N., Almeida, C., and Saraiva, J. (2016). Inovações tecnológicas em escolas públicas: análise de fatores motivadores. In Anais do Workshop de Informática na Escola, volume 22, page 291. 